\title{
An Update on Sudden Unexpected Infant Deaths - A Systematic Review on Aetiology, Reporting, Pathology and Medico Legal Aspects
}

\author{
Kulvinder Kochar Kaur ${ }^{1 *}$, Gautam Allahbadia ${ }^{2}$ and Mandeep Singh ${ }^{3}$ \\ ${ }^{1}$ Scientific Director, Dr Kulvinder Kaur Centre for Human Reproduction, Jalandhar, Punjab, India \\ ${ }^{2}$ Scientific Director, Rotunda-A Centre for Human Reproduction, Mumbai, India \\ ${ }^{3}$ Consultant Neurologist, Swami Satyan and Hospital, Jalandhar, Punjab, India \\ *Corresponding Author: Kulvinder Kochar Kaur, Scientific Director, Dr Kulvinder Kaur Centre for Human Reproduction, Jalandhar, \\ Punjab, India.
}

Received: September 03, 2019; Published: October 05, 2019

\begin{abstract}
SIDS (sudden infant death syndrome) is the leading cause of postneonatal infant mortality in the United States today, with an overall ratio of $0.39 / 1000$ live births. It is defined as the sudden and unexpected death of an infant $<12$ mth of age that remains unexplained after a complete autopsy, death scene investigation, and review of the clinical history. Despite significant decreases in incidence since the introduction of safe infant sleeping recommendations sudden infant death syndrome (SIDS) is still the major cause of neonatal death in western countries. Here we conducted a systematic review to find the aetiology of SIDS. Out of 11812 articles in pubmed search engine we selected 44 articles for this review. No meta-analysis was done. Various aetiological factors identified along with what are the methods that need to be adopted to reduce its occurrence are presented like the serotonin brain hypothesis, breastfeeding and sleeping position, air pollution with maternal consumption of caffeine and nicotine, leptomeningeal inflammation and iron, avoiding circumcision in prematurity, cardiac ion changes etc. are reviewed along with importance of forensic medicine to be able to find out the proper cause of the unexpected deaths in infancy.

Keywords: SIDS; SUDI; Serotonin Brain Hypothesis; Breastfeeding; Air Pollution (CO2); Leptomeningeal Inflammation; Prone Position and Breast Feeding; Autopsy
\end{abstract}

\section{Introduction}

Despite significant decreases in incidence since the introduction of safe infant sleeping recommendations sudden infant death syndrome (SIDS) is still the major cause of neonatal death in western countries. In the US, over 2500 infants die suddenly and unexpectedly each year with nearly 100 deaths annually in Australia. Health professionals play a key role in advicing parents how to make their infants sleep safely for minimizing the risk of sudden infant death syndrome and sleeping accidents. Infants should be placed supine to sleep in a cot with a firm well -fitting mattress in the parental bedroom with no soft or loose beddings that could obstruct the airway. Exposure to smoking both before and after birth should be minimized. Breastfeeding should be encouraged, as should immunization. Dummies can be recommended after Breastfeeding has been established. Thus Home 2019 reviewed what are the evidences behind these recommendations [1].

\section{Methods}

We conducted a systematic review using a pubmed search engine till 2019 using the MeSH terms like "sudden infant death syndrome", "sudden unexpected deaths in infancy", "causes of SIDS" air conditions", "sleeping positions", forensic aspect of SUDI investigation. 


\section{Results and Discussions}

We came across 11812 articles of which we selected 44 articles for this review. No meta-analysis was done.

\section{Aetiology}

Role of serotonin brain stem hypothesis

SIDS is the leading cause of postneonatal infant mortality in the United States (US) today, with an overall ratio of $0.39 / 1000$ live births. It is defined as the sudden and unexpected death of an infant $<12 \mathrm{mth}$ of age that remains unexplained after a complete autopsy, death scene investigation, and review of the clinical history. The serotonin brain stem hypothesis has been a leading hypothesis for SIDS in the last 2 decades. Kinney and Haynes laboratory has studied this hypothesis overtime with a variety of tissue techniques, including tissue receptor autoradiography, high performance liquid chromatography, Western Blot analysis, immunohistochemistry, and proteomics. They reviewed the progress in their laboratory toward supporting this hypothesis. They concluded that an important subset of SIDS infants have serotonergic abnormalities resulting from a "core lesion" in the medullary reticular formation comprised of the nuclei that contain serotonin neurons. This lesion could lead to a failure of protective brain stem responses to homeostatic challenges during sleep in a critical developmental period which causes sleep related sudden death [2].

Role of Air pollution along with nicotine and caffeine

Air pollution is well known as a potential risk factor for many diseases. A report on recent studies on air pollution exposure suggested that Air pollution also affects sudden infant death syndrome (SIDS), which is one of the leading causes of infant death during the $1^{\text {st }}$ year of life which strikes roughly one in every 1000 babies worldwide [3]. Though causes still remain unclear, the rebreathing of exhaled carbon dioxide $\left(\mathrm{CO}_{2}\right)$ trapped near an infant's airway has been suggested as a possible reason for the increased risk of SIDS [4]. $\mathrm{CO}_{2}$ has been understood to be an external stressor, and hence a proper air quality standard for daycare facilities is recommended. Due to this, children should not stay in a closed room during naptime to avoid high concentration of this gas. The National Institute of Occupational Safety and Health (NIOSH) guidelines suggests that $\mathrm{CO}_{2}$ concentrations in indoor air above $1000 \mathrm{ppm}$ is a clear indication of bad air, due to inadequate ventilation [5]. Recently, the combination of nicotine and caffeine has received considerable attention in view of their role in SIDS. Exposure of neonates to caffeine and nicotine is well known to be capable of affecting response to hypoxic conditions, that is a process believed to be related to SIDS [6]. These substances are present in large amounts in breast milk of women smoking and/or drinking coffee. Despite no recommended limits for these 2 substances are listed, it has been said that low doses $(<200 \mathrm{mg} /$ day) of caffeine can be consumed during breast feeding, the Food and Drug Administration has advised pregnant and lactating women to avoid caffeine -containing foods and drugs if possible. As far as nicotine is concerned the American Academy of Paediatrics no longer lists nicotine as a drug which is contraindicated in breast feeding [7].

However at the time of breast feeding, these toxic substances pass to the neonate, adding to the development of an underlying vulnerability in SIDS. Heavy caffeine intake was found to be significantly associated with an increased risk in a case control study [8]. Caffeine and nicotine alone might not be toxic enough for creating an underlying vulnerability for SIDS, but the combination of these factors are enough to put neonates at a higher risk of dying from SIDS [5].

These chemical substances have been mainly investigated for their side effects on human health. Nicotine is inhaled via cigarettes, while caffeine gets in the body by drinking coffee and soft drinks. These drugs do possess certain positive effects, like increased levels of acetylcholine in the brain [9], along with negative effects like increased BP, rapid breathing, heart palpitations, and insomnia in view of extended periods under their influence [10]. $\mathrm{CO}_{2}$ is a gas that is present in ambient fresh air at a concentration of about $380 \mathrm{ppm}$. But due to human and industrial activities and gas emissions from motor vehicles, these concentrations increase notably. Hence it is of interest to develop simple, low cost, small, and reliable $\mathrm{CO}_{2}$ sensors for monitoring this gas in ambient air [11].

Sudhan., et al. think that this is the $1^{\text {st }}$ report that has attempted to develop chemical sensors based on conductometric and electrochemical sensor platforms for monitoring these harmful substances. Among the variety of sensory sensors, those used here are popular, cheaper, and with the largest commercial appeal. Their functioning mechanism and application has been reported in many reviews and books [12]. Here they $1^{\text {st }}$ synthesized hydroxyapatite $\left(\mathrm{HA}-\mathrm{Ca}_{10}\left(\mathrm{PO}_{4}\right)_{6} \mathrm{OH}_{2}\right)$-graphene -multiwalled carbon nonotube nanocomposite (HA-GN-MWCNT) and used it as sensing material for i) modifying glass carbon electrode (GCE) for the electrochemi- 
cal determination of nicotine and caffeine, and ii) developing a conductometric $\mathrm{CO}_{2}$ gas sensor for monitoring indoor air quality (AQ).

Hydroxyapatite is one of the major inorganic nanoporous biomaterials, attracting many interesting applications in the biomedical field due to its excellent mechanical properties and good biocompatibility, so it has various potential applications in bone and teeth replacement. Also HA has been investigated as a sensing material for chemical and biochemical biosensors for both detection in liquid and gas environments $[13,14]$. The presence of $\mathrm{H}^{+}$and $\mathrm{OH}^{-}$ions is considered to be responsible for its ionic conductivity at room temperature, whereas oxygen ions $\left(\mathrm{O}_{2}^{-}, \mathrm{O}^{-}\right.$, etc) play a key role at higher temperatures. However for practical applications, conductivity of pure HA is not sufficiently high. A way to solve this limitation is to use HA composites, e. g. Hydroxyapatite in conjunction with organic conductive filters. Multiwalled carbon nanotubes (MWCNT) and graphene (GN) have been mainly used for this purpose in view of their positive mechanical properties, and increased electrical conductivity [15]. MWCNT are promising candidate materials for chemical and biochemical biosensors in view of their high surface area for gas adsorption, provided by central hollow core and outside walls. The reason graphene is used is due to the bearing of oxygen functional groups in edges and the basal plane of its honeycomb structure [16]. Combination of these 2 carbon materials offer a great flexibility and dispersion of MWCNT's on $\mathrm{CO}$, due to the presence of oxygen-containing functional groups in GO nanosheets. Additionally, this combination leads to an improvement in the electrical and electrochemical properties [17].

Combining these 2 carbon nanostructures with HA resulted in strong synergic effect and subsequently led to a robust and superior composite material with improved electrical and electrochemical properties and reduced agglomeration that sustains the stability of the nanometric HA phase. Sensing properties of chemical sensors based on temporary (hydroxyapatite-graphene - multiwalled carbon nonotube (HA-GN-MWCNT) nanocomposite in the detection of chemical substances representing risk factors for sudden infant death syndrome (SIDS), have been evaluated. Characterization data of the synthesized composite have shown graphene MWCNT network serves as a matrix to uniformly disperse the hydroxyapatite nanoparticles and provide suitable chemical properties needed for developing novel electrochemical and conductometric sensor. A HA-GN-MWCNT composite - modified glassy carbon electrode (HA-
GN-MWCNT/GCE) has been fabricated and tested for the simultaneous monitoring of nicotine and caffeine by cyclic voltammetry (CV) and square wave voltammetry ( (SWV), whereas a HA-GNMWCNT conductive gas sensor has been tested for the detection of $\mathrm{CO}_{2}$ in ambient air. Reported results pointed that the synergic combination of chemical properties of HA and electrical/electrochemical characteristics of the mixed graphene MWCNT network play a important role in increasing the electrochemical and gas sensing behavior of the tertiary HA-GN-MWCNT hybrid nanostructure. The high performance of the developed sensors make them suitable for monitoring unhealthy actions (eg smoking, drinking, coffee) in breastfeeding women and environmental factors (bad air quality), that are associated with increased risk of SIDS [18].

\section{Role of otoacoustic emission}

Despite its reduced incidence, sudden infant death syndrome (SIDS) is the second leading cause of postneonatal infant mortality in England and Wales [19], the highest among infants of younger mothers [20] and common in socially deprived families [21]. Epidemiological markers at birth is available to identify some families at increased risk [22] but are neither sensitive nor specific enough to make targeted intervention possible [23]. A physiological marker independent of current demographic identifiers would have the potential for making targeted intervention viable.

In 2008, a study demonstrated an altered newborn otoacoustic emission (OAE) hearing test in 31 infants who subsequently died of SIDS compared with 31 control infants [24]. The OAE is the sound signal generated by the cochlea as a response to sound and recorded in the ear canal. This observation begs the question -are altered OAE's a marker of predisposition to SIDS? If so, can the newborn OAE hearing test be used in combination with epidemiological markers to identify "at risk" infants at birth? However maternal smoking during pregnancy was not measured in the Rhodes Island Study, so it could be that exposure to tobacco smoke in utero resulted in developmental differences associated with infant hearing which would confound the potential of an improved screening tool but would still add to our understanding of causal mechanism s for some of these deaths. Most infants (> 99\%) in England have a hearing test shortly after birth and the data, sorted by Public Health England since 2010, could be suitable for analysis.

In England and Wales, SIDS deaths have fallen from a peak of nearly 1600 deaths in 1988 [25] to just over 200 deaths in 2016 
[20]. Longitudinal data collected in Avon suggest not only that there has been a shift towards poorer families but the prone positioning is still a feature of these deaths [21] and bed sharing in hazardous conditions (next to a parent who has consumed alcohol, drugs or regularly smokers or uses an unsafe sleeping surface such as sofa) has emerged as a highly significant risk factor $[22,26]$. Thus Blair., et al. conducted a retrospective case controlled study where telephonic interviews with families recruited in England between july 2016 and October 2017 who experienced the unexpected death of a child $<4$ yrs since 2008 and control families recruited from maternity wards in Bristol and Birmingham. They recruited 91 (89\%) of the 102 bereaved families who made initial contact, 64 deaths were under 1yr (sudden unexpected death in infancy) of which 60 remained unexplained (SIDS). Of the 220 control families, 194 (88\%) follow up interviews were conducted. They had analyzable data for 24 SIDS infants (40\%) and 98 controls (51\%). OAE signals were marginally increased rather than decreased among SIDS infants for the right ear, especially at lower frequencies, but not significantly so. The strongest predictors of SIDS were bed sharing in hazardous (infant sleeping next to a care who smoked, drank alcohol, or slept on a sofa) circumstances (35\% vs 3\% controls, p $<0.0001)$, infants found prone (33\%vs 3\%controls, $\mathrm{p}<0.0001)$ and infants whose health in the final weeks was 'not good' (53\% vs $9 \%$ controls, $p<0.0001$ ). The prevalence of maternal smoking during pregnancy among both SIDS mothers (20\%) and controls (10\%) was much lower than previous studies. Thus they concluded that hearing data were difficult to obtain; larger numbers would be needed to determine if asymmetrical differences between the right and left ear were a marker for SIDS. A national prospective registry for monitoring and a renewed campaign to a new generation of parents needs to be considered underlining the initial message to avoid bed sharing in hazardous circumstances [27].

\section{Role of Cardiac ion channel variants}

Paludan-Muller., et al. tried to investigate the pathogenicity of cardiac ion channel variants previously associated with SIDS. They reviewed SIDS-associated variants previously reported in databases and the literature in 3 large population-based cohorts; The ExAC database, theInter99 study, and the UK Biobank (UKBB). Variants were classified as per the American College of Medical Genetics and Genomics (ACMG) guidelines. Of the 92 SIDS -associated variants, 59 (64\%) were present in Ex AC. 18 (20\%) in Inter99, and $24(26 \%)$ in UKBB. Using the Inter99 cohort, they found no difference in J -point amplitude and QTc intervals between carriers and noncarriers for $14 / 18$ variants. There was no difference in the risk of syncope ( $p=0.32)$, malignant vascular arrhythmia $(p=0.96)$, and all cause mortality ( $p=0.59$ ) between carriers and noncarriers. The ACMG guidelines reclassified $75 \%$ of all variants as variant of uncertain significance, likely benign and benign. They identified $\sim 2 / 3$ of variants previously associated with SIDS and found no significant associations with ECG traits, syncope, malignant ventricular arrhythmia, or all cause mortality. These data indicated that these variants are not highly penetrant, monogenic causes of SIDS and underline the importance of frequent reappraisal of genetic variants to avoid future misdiagnosis [28].

\section{Role of SCN 10A Variant in cardiac changes}

Multiple genome -wide association studies (GWAS) and targeted gene sequencing have identified common variants in SCN10A in cases of PR and QRS duration abnormalities, atrial fibrillation and Brugada syndrome. The New York City Office of Chief Medical Examiner has now also identified 5 SCN10A variants of uncertain significance in 6 separate cases within a cohort of 330 sudden unexpected death events. The gene product of SCN10A is the Nav1. 8 sodium channel function to provide better information of these variants on Nav1. 8 sodium channel function to give better information to the reclassification of these variants. Patch clamp studies were done by Gando., et al. to assess effects of the variants on whole cell Nav 1.8 currents. They also performed RNA seq analysis and immuno fluorescence confocal microscopy to determine Nav 1.8 expression in heart. They showed that $4 / 5$ rare 'variants of unknown significance' (L388M, L867F and V 15181) are associated with altered functional phenotypes. The R756W variant behaved similar to wild type under their experimental conditions. They failed to detect Nav 1. 8protein expression in immuno fluorescence microscopy in rat heart. Moreover, RNA seq analysis failed to detect full length SCN10A mRNA transcripts in human ventricle or mouse specialized cardiac conduction system, suggesting that the effect of Nav 1.8 on cardiac function system is likely to be extra cardiac in origin. Thus they concluded that $4 / 5$ SCN10A variants of uncertain significance, identified in unexplained death, have deleterious effects on channel function. These data extend the genetic testing of SUD cases, but significantly more clinical evidence is required to satisfy the criteria needed to associate these variants with the onset of SUD [29].

Role of leptomenengeal inflammation and iron

Previous research shows that leptomeninges of infants and late term fetuses derived from nontraumatic, hospital -based co- 
hort contain a surprisingly large number of inflammatory cells and stainable iron. This was present irrespective of the findings from the general autopsy, the neuropathological examination, and the mode of delivery. Jack E., et al. applied a similar methodology to a sudden infant death syndrome/sudden unexpected death in infancy (SIDS/SUDI), 42 SIDS/SUDI cases autopsied between 2006-2014 by the San Diego County Medical Examiner's Office were identified. An interpretable amount of leptomeninges from at least 2 areas of the brain (cerebral cortex, brain stem, cerebellum) were present in each case. Immunoperoxidase (IPOX) staining with CD45 and CD68 was performed and Perl's method was used to detect the presence of iron. The number of immunoreactive cells per IPOX stain within the leptomeninges in each side was manually tabulated and the density subsequently quantified. The presence or absence of stainable iron was noted. The cohort represented 22 males and 20 females between the age from 2 to 311 days, with relatively evenly divided modes of delivery. The examined brain sections included 32 of the cerebral cortex, 18 of the brain stem, and 36 of the cerebellum. The lengths of the examined leptomeninges ranged from $2-40 \mathrm{~mm}$. The lengths of the examined ranged from 2-40 $\mathrm{mm}$. The ranges of the number of cells $/ \mathrm{ml}$, and the standard deviations of the mean were wide and varied. Overall there was no significant difference in the number of CD45 or CD68 immunoreactive cells/ $\mathrm{ml}$ between the 3 brain sites. Comparing the cohort to a subpopulation of hospitalized infants in their previous study, there was no significant differences between the density of inflammatory cells in the sections from the cerebral cortex and brain stem. There were differences in the CD68Bdensities, especially in the cerebellar sections, which might be attributable to methodological differences. Iron was identified in only a single section in the cohort but was present in most of the cases in the hospital based cohort. Thus they concluded that this study further examined the relevance of the presence of inflammatory cells and iron in the meninges. Whether the hospital based or more forensically relevant population, the presence of inflammatory cells in the leptomeninges (even in great abundance) is common [30].

\section{Role of circumcision and prematurity}

The allostatic load hypothesis posits that SIDS is the result of cumulative perinatal painful stressful, or traumatic exposures that tax neonatal regulatory systems. Elhalk 2019 aimed to test the predictions of the allostatic load hypothesis, for which they explored the relationship between SIDS and 2 common phenotypes, male neonatal circumcision (MNC) and prematurity. They collated latitu- dinal data from 15 countries and 20 US states sampled during 2009 and 2013. They used linear regression analyses and likelihood ratio tests to calculate the association between SIDS and phenotypes. SIDS mortality rate was significantly and positively correlated with MNC. Globally (weighted): increase of 0.06 (95\%CI: $0.01-0.16, \mathrm{t}$ $=2.81, \mathrm{P}=0.01)$ per 1000 SIDS mortality per $10 \%$ increase in circumcision rate. US (weighted): increase of 0.1 (95\%CI:0.03 - 0.1, T $=2.86, \mathrm{P}=0.01$ ) per 1000 unexplained mortality per $10 \%$ increase in circumcision rate. US states in which Medicaid covers MNC has significantly higher $\mathrm{MNC}$ rates $\left(\mathrm{X}^{-}=0.72\right.$ vs $\left.0.49, \mathrm{p}=0.007\right)$ and male/female ratio of SIDS deaths (X- $=0.1 .48$ vs $1.125 . \mathrm{p}=0.015$ ) than other US States. Prematurity was also significantly and positively correlated with MNC. Globally: increase of 0.5 (weighted: 95\%CI:0.02-0.086, $\mathrm{t}=3.37, \mathrm{p}=0.004$ ) per 1000 SIDS mortality per $10 \%$ increase in Prematurity rates. US Increase of 1.9 (weighted): 95\%CI: $0.06-0.32, t=3.13, p=0.004) 1000$ unexplained mortality per $10 \%$ increase in Prematurity rates. Combined the phenotypes increased the likelihood of SIDS. Thus they concluded that epidemiological analyses was useful to generate hypothesis but cannot provide strong evidence of causality. Biological plausibility is provided by a growing body of experimental and clinical evidence linking aversive preterm and early-life SIDS events. Together with historical and anthropological evidence their findings emphasize the necessity of cohort studies that consider these phenotypes with the aim of improving the identification of at-risk infants and reducing mortality. Thus parents need to understand that preterm birth and neonatal circumcision are associated with a greater risk of SIDS, and efforts should be focused on reducing their rates [31].

\section{Role of vaccination}

Sudden infant deaths might be attributable to side effect of vaccination, but separating them from coincidental occurrences is difficult. Osawa., et al. retrospectively investigated vaccination related details from postmortem findings of 57 cases of Sudden deaths in children 2yrs or younger. Data were extracted from autopsy files at the Department of Forensic medicine, Tokai University School of Medicine. Vaccination histories were available in 50 cases based on the maternity passbook. Of the 32 cases in which any vaccines was given, 7 infants $(21.9 \%)$ had received immunization before death was Haemophilus influenza B. Although a temporal association of vaccines with Sudden deaths was present for two-3mth old and one $14 \mathrm{mth}$ old infants in whom death occurred within 3 days of receiving the $H$. influenza type $b$ and other vaccinations, a definitive relationship between the vaccine and death could not be identified. 
Histopathological examinations revealed pneumonia and upper respiratory tract infections as contributing to death in their cases. Moreover all 3 cases showed hemophagocytosis in the spleen and lymph nodes, which are similar features of hemophagocytotic lymphohistiocytosis. Judgement of the disorders as truly related to vaccinationis difficult, but suspicious cases do exist. Forensic pathologist s must devote >attention to vaccination in Sudden infant death cases [32].

\section{Role of sleeping positions}

Prone sleeping position is the main known modifiable risk factor for SIDS. There are other SIDS recommendations although with less impact. Thus Ruiz-Botia., et al. aimed to describe the prevalence of Prone sleeping position along with other risk factors associated with SIDS in a sample of Spanish babies and infants. A cross-sectional study was carried out on 640 families with children from $0 \mathrm{mths}$ to $11 \mathrm{mths}$. In addition to the sleep association, the adherence to 4 other recommendations regarding SIDS was analyzed: place where infant sleeps, breast feeding, use of nonnutritive suction, and maternal smoking. A total of $41.3 \%$ of infants under 6 mths and $59.7 \%$ of infants aged 6to $11 \mathrm{mths}$ slept in a non recommended position. Only $6.4 \%$ of families analysed following all 5 recommendations. Thus there is a high prevalence of modifiable risk factors for SIDS among the studied population. Personalized education should be promoted, among other campaigns to raise awareness and prevent SIDS [33].

\section{Breast feeding and sleeping positions}

Breastfeeding and the place to sleep for the mother and the infant have been controversial internationally due to reported concerns regarding infant deaths despite the known benefits of exclusive and prolonged breastfeeding, which are increased by breastfeeding at night. Martinilli., et al. aimed this integrated analysis to i) review breastfeeding and maternal and infant sleep research literature via historical, epidemiological, anthropological and methodological lenses b) use this information to determine where we are currently in safeguarding both infant lives and breastfeeding knowledge and inform our policy and practice. Despite well meaning but unsuccessful campaigns in some countries to dissuade parents from sleeping with their babies, many breastfeeding mothers and caregivers do sleep with their infants whether intentionally or unintentionally. Taking cultural contexts and socioecological circumstances into consideration, data supports policies to counsel parents and caregivers on safe sleeping practices, in- cluding bed sharing in nonhazardous circumstances particularly in the absence of parental smoking, recent parental alcohol consumption, or sleeping next to an adult on a sofa. Further research with appropriate methodology is needed to drill down on actual rates of infants deaths, paying close attention to the definitions of death, the circumstances of the deaths, and confounding factors, in order to ensure we have the best information with which to derive public health policy, introduction and the use of the concept of "breast sleeping" is a plausible way to remove the negative connotations of "co-sleeping" and redirect ongoing data -driven discussions and education of best practices of breastfeeding and sleep [34].

Safe sleeping recommendations for infants have been evolving over the past 3 decades. It has been shown that physicians recommendations strongly influence parent's choice of infant sleep position. However the proportion of physicians and/or nurses giving infant sleep advice to parents is reportedly low. A survey was conducted in South Dakota by Angal., et al. to evaluate pediatricians ' and family practitioners ' knowledge of safe sleep recommendations for infants. Survey questions assessed their beliefs regarding risk factors for SIDS and their recommendations for safe sleep environments provided to parent/caregivers. Among the respondents $98 \%$ felt that it is important to discuss SIDS with every parent and $80 \%$ of them indicated a need within their profession to have further information on the topic of SIDS. However 31\% of physicians with 16 years or more since training and $64.5 \%$ of those with < than 16 years or more since training did provide safe sleep advice to parents and caregivers. Thus concluding that despite the knowledge of SIDS risk factors, gaps were seen in dissemination of information regarding all risk factors to parents. While sleep position, postnatal exposures and breastfeeding were more likely to be addressed, other elements of a safe sleep environment like bedding surface, bed sharing, pacifier use and room ventilation were less likely to be covered. These findings indicate the need for improved health care provider education/communication in South Dakota [35].

Unintentional suffocation is the $=>$ cause of injury death among infants $<1 \mathrm{yr}$ old in the US, with $82 \%$ being attributable to accidental suffocation and strangulation in bed. Understanding the circumstances surrounding these deaths might inform prevention strategies. Erck-Lambert., et al. analyzed data from the population based sudden unexpected infant death Case Registry from 2011 
to 2014. Cases categorized as explained suffocation with unsafe sleep factors (suffocation); these cases were more frequently attributed to soft bedding (69\%), followed by overlay (19\%) and wedging (12\%). Median age at death in mths varied by mechanism: 3 for soft bedding, 2 for overlay and 6 for wedging. Soft bedding deaths occurred most often in an adult bed (49\%), in a prone position (82\%), and with blanket (or blankets) obstructing the airway (34\%). Overlay deaths occurred most often in an adult bed (71\%) and infants were overlaid by the mother (47\%). Wedging deaths occurred most often when the infant became entrapped between a mattress and a wall (48\%). Thus concluding that safe sleep environments can decrease infant suffocation deaths. Increased knowledge about the characteristics of suffocation deaths can help inform prevention strategies by targeting highest-risk groups [36].

\section{Role of pathologists}

Despite being widely used few studies have assessed the utility of the San Diego SIDS. The purpose of this study conducted by Shipstone., et al. was to evaluate pathologists application of the San Diego definition in all cases of sudden unexpected death in infancy (SUDI) that occurred in Queensland. Australia between 2010 and 2014. Key coronial documents of 228 cases were reviewed independently by 3 reviewers and classified according to the San Diego definition. Clear guidance regarding the evidentiary threshold for classification and interpretation of the San Diego definition was provided. All reviewers classified cases identically in 202 cases $(88.6 \%)$. Consensus was achieved on the classification of the remaining 26 deaths following case discussion. After review, 79 cases were classified as SIDS, a one third reduction compared with the original classification, mainly due to a high probability of accidental asphyxia. The number of cases classified as undetermined (USID) almost doubled $(75 / 228,32.9 \%)$, and there was a $>$ than a 5 fold increase in cases classified as asphyxia $(43 / 228,18.9)$. Natural conditions reduced by approximately one third (21/228, 9.2\%). This study demonstrated that with clear guidelines for interpretation, the San Diego definition can be applied reliably with discrepancies involved through a process of peer review [37].

Role of registry for sudden unexpected deaths in infancy in England

The sudden and unexpected death of an infant child is devastating. An inability to explain why an infant or child died is difficult to accept for both families and professionals. No reliable national dataset exists to estimate precisely how many infants and children die unexpectedly each year in England. This lack of accurate epidemiological data belies the scale of this public health problem. Detailed controlled observational studies of infant deaths identifying risk factors and providing evidence based advice for parents has seen a dramatic reduction in incidence over the last $30 y e a r s$ by almost $80 \%$ but greater knowledge is needed if future deaths of infants and older children are to be prevented and families optimally supported. Thus Matthews., et al. proposed that a national registry of sudden unexpected deaths in infancy and childhood would accurately determine incidence, identify unknown risk factors and highlight good care practices, ensuring these can be standardized nationally. For such a project to be successful, however parents must be at the heart of it. They held a consultation day between families, professionals and supporting charities (The Lullaby Trust, Child Bereavement UK, SUDC UK and CRY) to seek opinion on the desire of a registry and the feedback they received from attendees regarding their views of the proposal and the practical aspects of administering it [38].

\section{Reporting of SUDI in Argentina}

Infant mortality comprises deaths among infants younger than one year old. The proportion of sudden unexpected death in infancy (SUDI) varies by country and based on the cause of the death. To describe the spatial and temporal variation of SUDI in Argentina between 1991 and 2014 according to the International Classification of Diseases, tenth revision. Based on infant death (provided by Health Statistics and Information Department) Chapur., et al. estimated the percentage of SUDI over the total number of infant deaths and the secular trend were estimated using a Poisson regression. The Sa TScan software, v 9.1.1 was used to detect clusters of districts where the percentage of SUDI was significantly different from the national percentage. In Argentina, between 1991and 2014, 267552 infants younger than 1 year died; 7\% corresponded to SUDI; the secular trend of causes was negative and statistically significant; the risk for SUDI was 0.86 , and a greater spatial heterogeneity was observed. At national level, the most common cause was SIDS, with interregional differences. In 9 district clusters, the risk of SUDI ranged between 4.36 and 1.24 , which is significantly different from the rest of the country. Thus they concluded that the proportion of SUDI and its causes show interregional heterogeneity; codes related to inaccurate diagnoses predominated in many un favourable regions, while SIDS was prevalent in the more developed regions [39]. 


\section{Medicolegal aspects of SIDS/SUDI}

Sudden unexpected death in infancy (SUDI) is the rapid and unexpected death of an infant aged less than 1year old. These deaths are referred for a medico-legal investigation to establish cause of death. National and regional protocols for the investigation of SUDI cases have been established in some countries and these typically include a death scene investigation component. However there is paucity of literature detailing the nature and extent of death scene investigation protocols. A systematic review was conducted to review the scope of death scene investigation of SUDI cases worldwide. Relevant articles ( $n=74)$ were identified by searching four literature databases, 3 discipline specific journals, as well as each articles reference list, until saturation was reached. Of the 16 countries represented by the included articles, 7 made specific reference to the standardized protocol used, which included photography, interview and/or scene re-enactment, investigation which varied between countries, and between SUDI admissions. This may be attributed to the differing socio-economic standings of the countries, and the resources available. Only 4 studies were from developing countries, 3 of which originated from South Africa. Overall investigation; as such, death scene investigation should be encouraged in SUDI investigation. To this end, protocols should be established nationally, and contain core analysis, which could be expanded depending on the needs and resources of the country [40].

The forensic pathologists responsible for sudden unexpected death (SUD) investigation in the pediatric setting faces many challenges. It usually takes many years to obtain reasonable experience and exposure to the wide variety of diseases that may present as SUD investigation in a pediatric context, and to appreciate the differences in the etiology and clinical context between the pediatric and adult SUD setting. In pediatric SUD, it is necessary to conduct a systematic, pediatric -focused autopsy investigation including extensive histopathological assessment and ancilliary testing. Postmortem histological findings in the context of SUD in pediatric population are often subtle and distinctly different from those seen in adult population. The pathologist must have an understanding of both developmental and pathological processes in order to correctly interpret the findings during a pediatric autopsy. A system based, histopathology -focused review of common entities, normal variants, and incidental findings that can prove challenging was discussed by D'Arcy., et al. For the forensic pathologist tasked with pediatric SUD autopsies, development of strong collaborative rela- tionship with a pediatric pathologist and/or neuropathologist to assist with histopathological analysis is strongly endorsed [41].

In a secondary analysis of publicy available data, the rate of sudden unexpected infant death in the 1st year exceeded the peak rates of the leading causes of injury death of youth $(<=19$ years of age). The absolute risk of sudden unexpected infant death exceeded age specific risks of the major causes of child injury mortality regardless of race or ethnicity [42].

Less invasive perinatal and paediatric autopsy methods, such as imaging alongside targeted endoscopy and organ biopsy, may address declining consent rates for traditional autopsy, but their accessibility and accuracy are not known. Lewis., et al. aimed to give empirical data on the acceptability and likely uptake for different types of autopsy among key stakeholders (study 1); and to analyze existing autopsy data sources to provide estimates of potential efficiency of less invasive autopsy (LIA) and its projected utility in clinical practice (study 2). Study1: was a mixed methods study. Parents were involved in research design and interpretation of findings. Substudy1: a cross sectional survey of 859 parents who had experienced miscarriage, termination of pregnancy for fetal anomaly, still birth, infant or child death, and interviews with 20 responders. Sub study 2: interviews with 25 health professionals and 4 coroners. Substudy3: interviews with 16 religious leaders and 8 focus groups, with 76 members of the muslim and jewish community. Study 2: a retrospective analysis of national data in addition to detailed information from an existing in -house autopsy databases of $>5000$ clinical cases that had undergone standard autopsy to determine the proportion of cases by clinical indication group for tissue sampling of specific internal organs significantly contributed to the diagnosis. In Substudy1: $91 \%$ of participants indicated that they would consent to some form of LIA, 54\% would consent to standard autopsy, 74\% of minimally invasive autopsy (MIA) and 77\% to non-invasive autopsy (NIA), Substudy 2: participants viewed LIA as a positive development, but had concerns around the limitations of the technology and deskilling the workforce. Cost implications, skills and training requirements were identified as implementation challenges. Substudy 3: religious leaders agreed that NIA was religiously permissible, but MIA was considered less acceptable. Community members indicated that they might consent to NIA if the body could be returned for burial with 24 hours. Study2: in $5-10 \%$ of cases of Sudden unexplained 
death in infants and Sudden unexplained death in childhood, the final cause of death is determined by routie histological sampling of macroscopically normal organs, predominantly the heart and lungs, and in this group routine histological sampling therefore remains an important aspect of investigation. In contrast routine histological examination of macroscopically normal organs rarely $(<0.5 \%)$ provides the cause of death in fetal case, making LIA and NIA approaches potentially highly applicable. A key limitation of the empirical research is that it is hypothetical. Further research is needed to determine actual uptake. Furthermore because of the retrospective nature of the autopsy data set, findings regarding the likely contribution of organ sampling to final diagnosis are based on extrapolation of findings from historical autopsies, and prospective data collection is needed to validate the conclusions. Thus they concluded that LIA is viable and acceptable (except for unexplained deaths), and likely to increase uptake. Further health economic performance and implementation studies are needed to determine the optimal service configuration needed to offer this as routine clinical care. In plain language Autopsy (postmortem) examination of babies and children who die is often necessary to help drs or coroners to find out the cause of death. It might be useful for research as well. However many bereaved parents dislike the idea of their child being cut and some religious communities prohibit the procedure. Over the past 30 yrs, consent rates for autopsies have declined. To address the parental concerns and declining uptake, a number of less invasive options have been developed. These include X-Ray, and magnetic resonance imaging (MRI), by doing keyhole internal examination and needle organ biopsy. However it is not known to what extent such methods are acceptable to parents, nor how accurate they are. Lewis., et al. surveyed the attitudes of bereaved parents and religious group leaders to such less invasive methods. The less invasive options was considered acceptable and would be chosen by almost 1000 bereaved parents. Such an approach is also acceptable to those religious groups for whom standard autopsy examination is not. They also examined a database of $>5000$ standard autopsies, tradional organ biopsy contributed to the diagnosis. In > 5000 standard autopsies, tradional organ biopsy contributed to determination of the cause of death or the main diagnosis. Hence a more limited and targeted tissue sampling protocol could be introduced without significant reduction in the accuracy of final diagnosis. The specific approaches circumstances and are likely to include a range, from targeted organ biopsy with an open incision, through incisionless image-guided needle biopsies, to noninvasive imaging only technique. Future studies may focus on how the NHS could implement offering less invasive approaches nationally, what the cost-benefit of such an approach could be and what the impact could be on real world uptake if this were to be offered routinely [43].

When an infant dies suddenly and unexpectedly, it is critical to correctly determine if the death was caused by child abuse or neglect, sudden unexpected infant deaths should be comprehensively investigated, ancillary tests and forensic procedures should be used to more-accurately identifying the cause of death, and parents deserve to be approached in a nonaccusatory manner during the investigation. Missing a child abuse death can place other children at risk, and inappropriately approaching a sleep-related death as maltreatment can result in inappropriately approaching a sleep related death as maltreatment can result in inappropriate criminal and protective services investigation. Communities can learn from these deaths by using multidisciplinary child death reviews. Paediatricians can support families during investigation, and advocate for establishing comprehensive and fully funded child death investigation and reviews at the local and state levels. Additional funding is also needed for research to advance our ability to prevent these deaths [44].

\section{Conclusion}

Thus in this review we have tried to emphasize on the various aetiological factora for SIDS/SUDI which include serotoninergic brain hypothesis. air pollution with high $\mathrm{CO}_{2}$ along with nicotine and caffeine intake, affect of changes in cardiac ions, leptomeningeal inflammation and iron, role of vaccination, circumcision especially in premature neonates and infant sleeping position, bedding nature. Further importance of reporting in all countries is important. Since autopsy is usually preferred by parents of these infants variety of methods to find the cause of this unexplained phenomena less invasive techniques have been suggested like $\mathrm{X}$ ray and MRI, Needle biopsy and endoscopy rather than standard autopsy that is acceptable to parents along with religious groups. Thus taking care of environment in which child sleeps, with good ventilation, avoiding of nicotine and caffeine by mothers, breastfeeding and avoid prone postures and separate bedding for child and adults and precautions during vaccination are some ways to prevent SIDS/SUDI. 


\section{Bibliography}

1. Home RSC. "Sudden infant death syndrome: current perspectives". Internal Medicine Journal 49.4 (2019): 433-438.

2. Kinney HC and Haynes RL. "The Serotonin Brain stem Hypothesis for the Sudden infant death syndrome". Journal of Neuropathology and Experimental Neurology 78.9 (2019): 765-779.

3. Dales R., et al. "Air Pollution and Sudden infant death syndrome". Paediatrics 113.6 (2004): 628-631.

4. Ferrig SF and LeeLW. "Indoor air quality assessment of day care facilities with carbondioxide, temperature and humidity as indicators". Journal of Environmental Health 65.4 (2002): 14-18.

5. Indoor Environmental Quality: Building Ventilation National Institute for Occupational Safety and Health. Washington, DC, USA (2011).

6. Adgent MA. "Environmental tobacco and Sudden infant death syndrome. A review". Birth Defects Research Part B 77.1 (2006): 69-85.

7. Calvaresi V., et al. "Transfer of nicotine, cotinine and caffeine into breast milk in a smoker mother consuming caffeine and drinks". Journal of Analytical Toxicology 40.6 (2016): 473-477.

8. Ford RPK., et al. "Heavy Caffeine intake in pregnancy and Sudden infant death syndrome". Obstetrical and Gynecological Survey 53 (1998): 535-536.

9. Conger SA., et al. "Does Caffeine added to Carbohydrate Provide Additional Ergogenic Benefit of Endurance?" International Journal of Sport Nutrition and Exercise Metabolism 21.1 (2011): 71-84.

10. Lee HJ., et al. "Molecular absorption study of nicotine and caffeine on single walled carbon nanotubes from first principles". Chemical Physics Letters 580 (2013): 57-61.

11. Van Hieu N., et al. "Comparative study on $\mathrm{CO} 2$ and $\mathrm{CO} 2$ sensing performance of La OCl-coated $\mathrm{Z} \mathrm{nO}$ nanowires". Journal of Hazardous Materials 244 (2013): 209-216.

12. Nert G. "First fifty years of Chemo resistive Gas Sensors". Chemo Sensors 3 (2015): 1-20.

13. Zhang Y., et al. "Green electrochemical sensing platforms utilizing hydroxyapatite derived from natural fish scales as a novel electro chemical material for the sensitive detection of kidney injury molecule 1 (KIM1)". Analyst 139 (2014): 536.
14. Lu L., et al. "A novel tyrosinase biosensor based on hydroxyapatite -chitonan nanoconposite for the detection of phenolic compounds". Analytica Chimica Acta 665 (2010): 146-151.

15. Khomenko V., et al. "Optimization of an asymmetric manganese oxide/activated carbon capacitator working2 $\mathrm{V}$ in aqueous medium". Journal of Power Sources 153 (2016): 183-190.

16. Petit C., et al. "Revisiting the chemistry of graphite oxides and its effects on ammonia adsorption". Journal of Materials Chemistry 19 (2009): 2176.

17. Aboutalebi SH., et al. "Comparison of GO, GO/MWCNTs composite and MWCNTs as potential electrode materials for supercar pacitators". Energy and Environmental Science 4 (2011): 1855.

18. Sudhan N., et al. "Monitoring of Chemical Risk Factors for Sudden infant death syndrome (SIDS) by hydroxyapatiteGraphene-MWCNT Composite-Based Sensors". Sensors 19 (2019): 3437.

19. Office for National Statistics. Child mortality in England and Wales (2016).

20. Office for National Statistics. "Statistics bulletin: unexplained deaths in infancy". England and Wales: (2016).

21. Blair PS., et al. "Major changes in the epidemiology of Sudden infant death syndrome: a 20 year population based study of all unexpected deaths in infancy". Lancet 367.9507 (2006): 314-319.

22. Blair PS., et al. "Hazardous cosleeping environments and risk factors amenable to change: case control study of SIDS in south west England". BMJ 333 (2009): b3666.

23. Fleming PJ., et al. "Sudden unexpected deaths in infancy: aetiology, pathophysiology, epidemiology and prevention in 2015". Archives of Disease in Childhood 100.10 (2015): 984988.

24. Rubens DD., et al. "Newborn oto-acoustic emission hearing screening test: preliminary evidence for a marker of susceptibility to SIDS". Early Human Development 84 (2008): 225-229.

25. Gilbert R. "The changing of epidemiology of SIDS". Arch Dis Child 70.5 (1994): 445-449.

26. Blair PS., et al. "Bed sharing in the absence of hazardous circumstances: is there a risk of Sudden infant death syndrome? An analysis from two-case control studies conducted in the UK". PLoS One 9 (2014): e107799. 
27. Blair PS., et al. "Sudden infant death syndrome (SIDS) and the routine otoacoustic emission infant hearing screening test: an epidemiological retrospective case-control study". BMJ Open 9.7 (2019): e030026.

28. Paludan-Muller C., et al. "Reappraisal of variants previously linked with Sudden infant death. syndrome: results from three population-based cohorts". European Journal of Human Genetics 27.9 (20198): 1427-1435.

29. Gardo I., et al. "Functional characterization of SCN 10A variants in several cases of sudden unexpected death". Forensic Science International 301 (2019): 289-298.

30. Jack E., et al. "Evaluation of the presence and distribytion of leptomeningeal inflammation in SIDS/SUDI with a hospital based cohort". Child's Nervous System (2019).

31. Elhaik E. "Neonatal circumcision and prematurity are associated with Sudden infant death syndrome". Journal of Clinical and Translational Research 4.2 (2019): 136-151.

32. Osawa M., et al. "Sudden infant death After Vaccination: Survey of Forensic Autopsy Files". The American Journal of Forensic Medicine and Pathology 40.3 (2019): 232-237.

33. Ruiz-Botia I., et al. "Sudden infant death syndrome: Do the parents follow the recommendations?". and Paediatr (Barc) (2019): S1695-4033.

34. Marinelli KA., et al. "An Integrated analysis of Maternal -Infant Sleep, Breastfeeding, and Sudden infant death syndrome Research Supporting a Balanced Discourse". Journal of Human Lactation 35.3 (2019): 510-520.

35. Angal J., et al. "Physicians knowledge and Practice of Safe Sleep Recommendations for infants in south Dakota". SD Med 72.8 (2019): 439-453.

36. Erck-Lambert AB., et al. "Sleep-Related Infant Suffocation Deaths Attributable to Soft Bedding, Overlay, and WEDGING". Pediatrics 143.5 (2019): e20183408.

37. Shipstone RA., et al. "An evaluation of pathologists' application of the diagnostic criteria from San Diego definition of SIDS and unclassified Sudden infant death". International Journal of Legal Medicine (2019).

38. Mathews E., et al. "National registry for sudden unexpected deaths of infant and children in England :why do we need one and do families want one?" Archives of Disease in Childhood (2019).
39. Chapur VF., et al. "Epidemiology of sudden unexpected deaths in infancy in Argentina: secular trend and spatial variation". Arch Argent Paediatr 117.3 (2019): 164-170.

40. Bennett T., et al. "Global trends in the extent of death scene investigation performed for sudden and unexpected death of infant (SUDI) cases: A systematic review". Forensic Science International 301 (2019): 435-444.

41. D’Arcy C., et al. "Histopathologic analysis in sudden infant and child deaths: A practical approach". Academic Forensic Pathology 8.3 (2018): 492-538.

42. Roehler DR Batra EK and Quinlan KP. "Comparing the risk of sudden unexpected infant deaths to Common Causes of Childhood injury Death". The Journal of Pediatrics 212 (2019): 224227.

43. Lewis C., et al. "Minimally invasive autopsy for fetuses and children based on a combination of postmortem MRI and endoscopic examination: a feasibility study". Health Technology Assessment 23.46 (2019): 23 (46): 1-104.

44. Palusci VJ., et al. "Identifying child abuse Fatalities during Infancy". Paediatrics 144.3 (2019): e20192076.

Volume 2 Issue 11 November 2019 (C) All rights are reserved by Kulvinder Kochar Kaur., et al. 